\title{
Projeto de Automação e Controle: Controle de Sistemas a Eventos Discretos para Posicionamento de Painéis Solares
}

\author{
José Eduardo Rabelo e Rabelo* Ernesto Marçal Ferreira Franklin** Anna Dulce Espindola Fonseca Rocha** \\ João Viana da Fonseca Neto *
}

\author{
* Laboratório de Sistemas Embarcados e Controle Inteligente - LABSeci, Departamento de Engenharia Elétrica, Universidade \\ Federal do Maranhão - UFMA ( e-mail: eng.eduardo.rabelo@gmail.com/jviana@dee.ufma.br). \\ ** Instituto Federal do Maranhão - IFMA (e-mail: \\ ernesto.ferreira@ifma.edu.br/anna.rocha@ifma.edu.br)
}

\begin{abstract}
This work proposes the automation of the movement system for solar panels using systems control techniques to discrete events embedded in Programmable Logic Controllers, for application in a solar panel model developed in the Laboratory of Control and Automation of the Federal University of Maranhão with the intention of positioning the panel in function of the displacement of the sun, aiming at a gain in the generation of energy comparatively when the panel is fixed. Initially an analysis will be made in the theory of Systems to Discrete Events and in the solar panel model and equations. Then the construction of the elements that constitute the project will be demonstrated. At the last moment will be shown and discussed the results obtained from the simulations performed when the panel remains fixed at $90^{\circ}, 45^{\circ}$ and when the proposed control are implemented.
\end{abstract}

Resumo: Este trabalho propõe a automação do sistema de movimentação para painéis solares utilizando às técnicas de controle de sistemas à eventos discretos embarcados em Controladores Lógicos programáveis, para aplicação em um modelo de painel solar desenvolvida no Laboratório de Automação e Controle da Universidade Federal do Maranhão com a intenção de posicionar o painel em função do deslocamento do sol, visando assim um ganho na geração de energia comparativamente quando o painel está fixo. Inicialmente será feito uma análise na teoria de Sistemas à Eventos Discretos e no modelo e equações do painel solar. Em seguida serão demostrados a construção dos elementos que constituem o projeto. No último momento serão mostrados e discutidos os resultados obtidos das simulações realizadas quando painel permanece fixo à $90^{\circ}, 45^{\circ}$ e quando o controle proposto for implementado.

Keywords: Solar Panels, Embedded systems, Programmable logic Controllers, Power generation, Systems to Discrete Events.

Palavras-chaves: Painéis Solares, Sistemas Embarcados, Controladores lógicos programáveis, Geração de energia, Sistemas à Eventos Discretos.

\section{INTRODUÇÃO}

Energia sustentável ou energia limpa são termos comumente falados em conferências mundiais ou por pesquisadores da área, isso em virtudes dos desafios relacionados ao tema em um futuro a curto, médio e longo prazo. Por energia sustentável entende-se como a necessidade em garantir uma oferta de energia adequada para gerações futuras não esquecendo da integridade fundamental dos sistemas naturais essenciais, bem como expandir a geração de modo a atender as mais de 2 bilhões de pessoas em todo mundo que atualmente não tem acesso às modernas formas de energia. (Goldemberg, 2004)

Destacam- se como fontes de energia utilizadas no mundo: hidroelétrica, solar, eólica, biomassa, combustíveis fosseis, energia nuclear, energia geotérmica e energia das marés. Galdino explica que:
"Dentre todas essas fontes, a energia nuclear e a dos combustíveis fosseis são consideradas não renováveis, pois os processos de sua utilização são irreversíveis e geram resíduos prejudiciais ao meio ambiente. As demais são consideradas renováveis e limpas, pois não consomem combustíveis e não produzem resíduos prejudiciais, e, quando bem planejadas, não geram consequências para o meio ambiente" (Galdino, 2000)

A energia solar vem sendo nos últimos anos de grande interesse por parte dos acadêmicos e empresários em função da sua perspectiva promissora em se poder gerar mais energia com valores menores. Em geral, a energia solar consiste na captação da radiação solar e a sua devida utilização, como por exemplo: aquecimento de água ou diretamente com eletricidade. 
O efeito fotovoltaico, como é chamado o aparecimento de uma diferença de potencial nas extremidades de um material semicondutor em função da luminosidade, pode ser observado em pequenas células solares com aparecimento de uma tensão entre seus terminais, a distância da fonte luminosa determinará o valor da tensão gerada (Rabelo, 2017).

A utilização da energia solar atualmente é possível através dos módulos fotovoltaicos que na prática são os agrupamentos de pequenas células ligadas em série ou em paralelo. Assim, com o intuito de potencializar a geração da energia grandes parques solares são fabricados onde vários módulos são organizados em serie ou em paralelo formando grandes arranjos fotovoltaicos.

A análise das características e o comportamento dos módulos fotovoltaicos levaram os pesquisadores a traçarem curvas que demonstram a relação da radiação solar com a tensão e corrente gerada nos terminais dos módulos. Com isso, foi observado que a partir da construção o módulo teria parâmetros como: corrente de curto circuito, tensão de circuito aberto além disso, estas curvas mostraram a dinâmica da geração e permitiram encontrar pontos de particular interesse como: tensão máxima, corrente máxima, e por conseguinte a potência máxima. Estes pontos máximos revelam a eficiência da geração e do efeito fotovoltaico a partir da radiação solar ou de uma fonte luminosa.

Algumas aplicações de módulos solares utilizam eles de forma fixa nos pontos de captação da radiação, com isso sua eficiência e geração diminuiu em função do deslocamento solar, ou seja, parte da radiação não é convertida em eletricidade.

Para otimizar a geração a partir da energia solar, muitas técnicas de controle aliadas a inteligência artificial com o auxílio do conhecimento do comportamento do sol possibilitaram que estes módulos a partir de estruturas próprias movimentem-se em busca de um ponto em que a geração de energia fosse maior (Rabelo, 2017). Assim, as literaturas apresentam essas técnicas ou mecanismos como algoritmos de busca pelo ponto de máxima potência nos painéis solares.

O presente artigo pretende demonstrar um projeto de automação e controle a partir das técnicas de controle de sistemas a eventos discretos com várias entradas que captam a radiação solar e posicionam o painel de modo a comparar a geração quando o painel permanece fixo à $90^{\circ}$, à $45^{\circ}$ e quando a lógica de controle inteligente implementada for utilizada.

O capítulo 2 demonstrará a fundamentação teórica que possibilitou a implementação da lógica de controle proposta. O capitulo 3 apresentará as partes constituintes do projeto e sua organização, como também o fluxograma do algoritmo utilizado. O capitulo 4 avaliará os resultados obtidos com o experimento realizado.

\section{AUTOMAÇÃO E SISTEMAS FOTOVOLTAICOS}

\subsection{Sistemas de Eventos Discretos}

A palavra automação tem sua origem no grego ( $g r$ autómatos) que significa movimentar-se por ou o que se movimenta sozinho. A base tecnológica para a realização da automação é o controle automático, as contribuições da engenharia para a execução deste controle estão divididas para melhor entendimento nas especialidades de controle que são: o controle dinâmico e o controle lógico. Pode-se ainda classificá-las em: Controle de Sistemas a variáveis contínuas (SVC) e Controle de Sistemas a Eventos Discretos (SED).

O controle de SED, segundo (Miyagi, 2001), é baseado num procedimento pré-estabelecido ou numa lógica fixa que estabelece um procedimento, executando ordenadamente cada estágio de controle. (Morais e Castrucci, 2013) comenta que este tipo de controle tem por objetivo complementar sistemas lógicos de maneira que eles respondam eventos externos ou internos de acordo com novas regras que são desejáveis de um ponto de vista utilitário.

Matematicamente, o controle de sistemas a eventos discretos pode ser mostrado da seguinte forma:

$$
\begin{gathered}
z(k+1)=h\{z(k), v(k)\} \\
w(k)=q\{z(k), v(k)\}
\end{gathered}
$$

Sendo:

- $v$ representa o vetor dos sinais de entradas de atuação sobre os $m$ elementos estruturais;

- $\quad z$ representa o vetor dos $n$ estados dos $m$ elementos estruturais;

- $\quad w$ representa uma variável vetorial de saída que é uma combinação dos $m$ elementos estruturais e $n$ estados assumidos por estes; e

- $\quad h\{$.$\} e q\{$.$\} São funções matriciais.$

A Fig. 1 mostra o modelo do objeto de controle do sistema de eventos discretos e a Fig. 2 ilustra o comportamento do mesmo em diagramas de blocos,

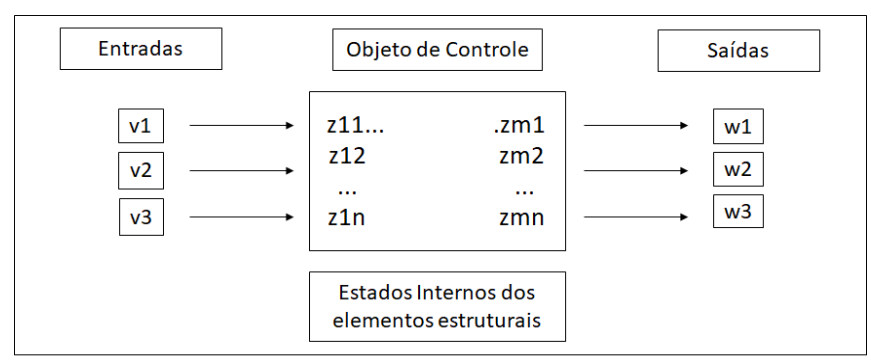

Fig. 1 Modelo do Objeto de Controle do Sistema a Eventos Discretos.

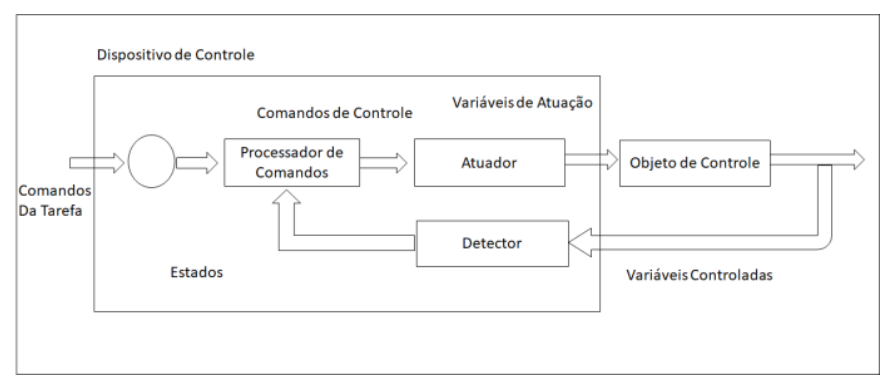


Fig. 2 Sistema de Controle SED

\subsection{Efeito Fotovoltaico}

O efeito fotovoltaico, como é conhecido a conversão direta da luz em eletricidade foi observado pela primeira vez pelo físico francês Edmond Becquerel, em 1839. O físico de 19 anos, comenta Masters "conseguiu fazer aparecer uma tensão quando ele iluminou um eletrodo de metal em uma solução de eletrólito fraco". (Masters, 2004). Quase 40 anos depois, prossegue Masters "Adams e Day foram os primeiros a estudar o efeito fotovoltaico em sólidos" (Masters, 2004)

A energia fotovoltaica utiliza materiais semicondutores para converter a luz solar em eletricidade. A tecnologia para fazer esta conversão está intimamente relacionada com as tecnologias de estado sólido utilizadas na construção de transistores, diodos.

Quando a energia solar (fótons) atinge a célula solar, os elétrons são soltos dos átomos no material semicondutor, criando pares de elétrons-buracos. Se os condutores elétricos estiverem conectados aos lados positivo e negativo, formando um circuito elétrico, os elétrons são capturados sob a forma de corrente elétrica, chamada fotocorrente. (Kalogirou, 2009)

O circuito equivalente de uma única célula pode ser visualizado na Fig. 3,

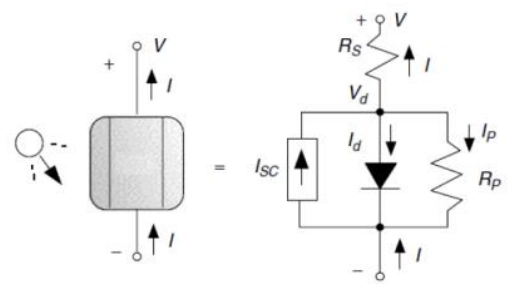

Fig. 3 Circuito equivalente de uma célula fotovoltaica. Fonte: Masters (2004)

A análise do circuito equivalente de uma célula fotovoltaica a partir das leis que regem a eletricidade permite a obtenção da equação que descreve o comportamento da célula, conforme a seguir:

$$
I=I_{s c}-I o\left[e^{38.9(V+I * R s)}-1\right]-\frac{1}{R p} *(V+I * R s)
$$

Esta equação possibilita a plotagem da Curva $I * V$ que mostra a dinâmica da captação, isto permite encontrar os pontos de particular interesse nos estudos de energia fotovoltaica, a Fig. 4 mostra o gráfico da curva $I * V$.

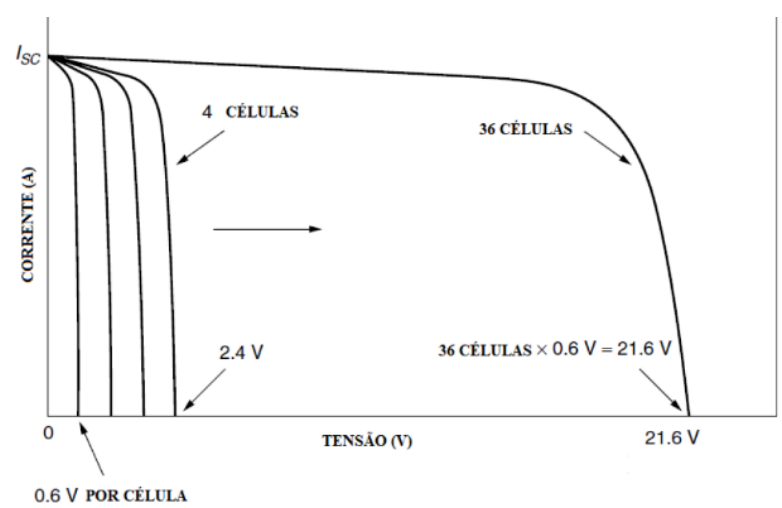

Fig. 4 - Curva $I * V$ para módulos fotovoltaicos.

A Fig. 4 ilustra a dinâmica do efeito fotovoltaico, a corrente de curto circuito é representada por $I_{s c}$ e a tensão de circuito aberto é aquela mostrada no eixo das ordenadas, a análise do gráfico é a seguinte: para uma única célula tem-se o valor de tensão de circuito aberto de $0.6 \mathrm{~V}$ para uma dada corrente $I_{s c}$, aumentando-se o número de células, neste caso para 36, ligando-as em série, maximiza o valor da tensão para 21.6V mantendo - se a corrente a mesma, o joelho da curva, é o que se chama na literatura de ponto de máxima potência. É neste ponto, para as condições da construção do módulo, a máxima tensão e corrente são obtidas, e por conseguinte, a máxima potência é alcançada, significando que neste ponto, o painel está gerando a partir da energia solar o seu máximo de eletricidade.

\section{PROJETO DE CONTROLE SED PARA POSICIONAMENTO DE PAINÉIS SOLARES}

O presente artigo propõe o controle SED para posicionamento de painéis solares visando encontrar a maior potência em relação ao painel fixo, a partir da medição direta da radiação solar através de pequenas células solares localizadas na superfície do painel. O diagrama de blocos da Fig. 5, mostra os elementos que constituem o projeto e como estarão dispostos.

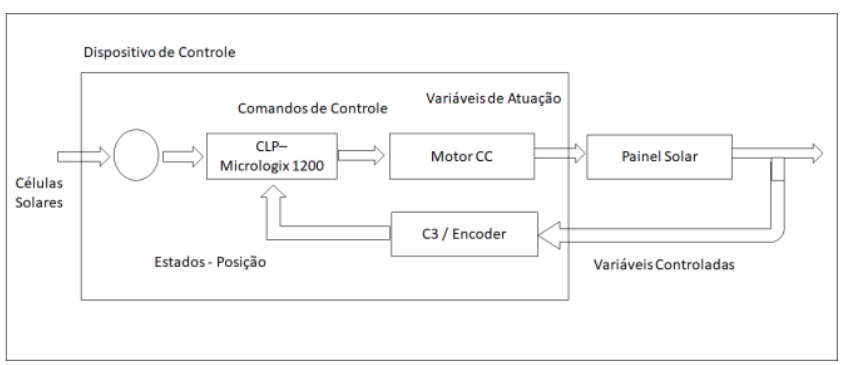

Fig. 5 Diagrama de Blocos do Projeto

O diagrama de blocos apresentado na Fig. 5 mostra os elementos que constituem o projeto. Inicialmente, os comandos de tarefa são as células solares que captarão a informação em relação a radiação solar. As informações provenientes das células são recebidas e processadas no CLP Micrologix 1200, a partir do processamento dos dados o Motor CC será acionado deslocando o painel para a posição desejada, possibilitando o controle inteligente do painel a partir do CLP (Fonseca, 2013). O motor CC será parado assim que o painel atingir a posição desejada a partir do comando de tarefa, a posição atingida será salva. Definiu-se que o painel teria uma atuação de meio círculo, ou seja, de $180^{\circ}$. Sendo que o caminho percorrido pelo painel se dará de $30^{\circ}$ a $150^{\circ}$.

A execução lógica do movimento do painel se dá através de comparações dos valores lidos e processados a partir das células solares colocados na superfície do painel solar. A Fig. 6 ilustra o fluxograma da lógica utilizada. 


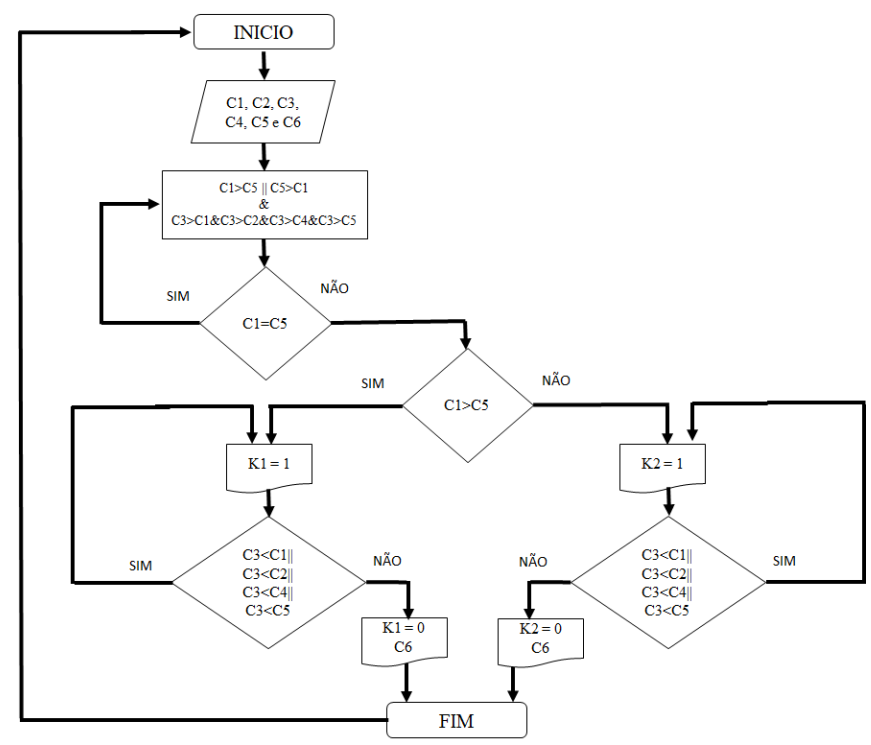

Fig. 6 Fluxograma do algoritmo utilizado.

O presente projeto está disposto e agrupado com a utilização: 1 - Painel Solar; 2 - Motor CC; 3 - CLP Micrologix 1200; 4 - Encoder; e 5 - Pequenas células solares. As ligações e montagens podem ser visualizadas nas Fig. 7 e 8.

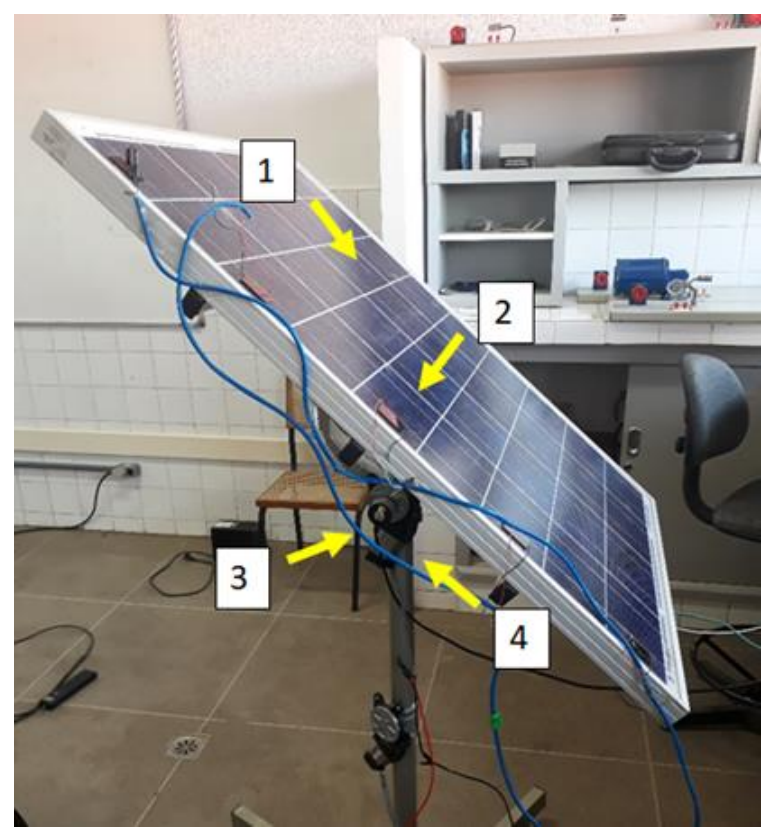

Fig. 7 Planta de Utilização

Legenda:

1. Painel Solar

2. Células Solares

3. Encoder Rotativo

4. Motor CC

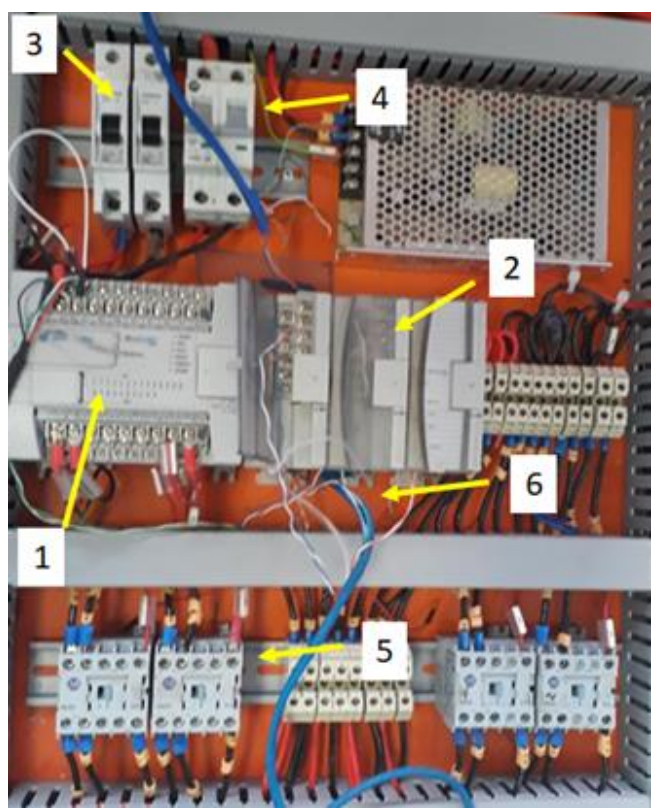

Fig. 8 Ligações das E/S do CLP

Legenda:

1. Micrologix 1200 - Ranhura 0

2. Entradas Analógicas

3. Disjuntor CLP

4. Disjuntor MOTOR

5. Contatores

Sinais de Entradas (Sinais das Células).

\section{AVALIAÇÃO DO SISTEMA DE CONTROLE}

Para avaliação do sistema de controle implementado foi necessário um experimento para a movimentação de uma fonte luminosa (uma lâmpada) em torno da placa solar. Isso se deu pelo fato das condições inviáveis de deslocamento e permanência do painel em um ambiente externo. Os resultados obtidos neste experimento foram realizados no dia 04/12/2017 por volta das 17:30 horas no Laboratório de automação e controle da Universidade Federal do Maranhão.

A Fig. 9 ilustra o procedimento utilizado e a Fig. 10 apresenta a maneira que a leitura da saída do painel foi lida.

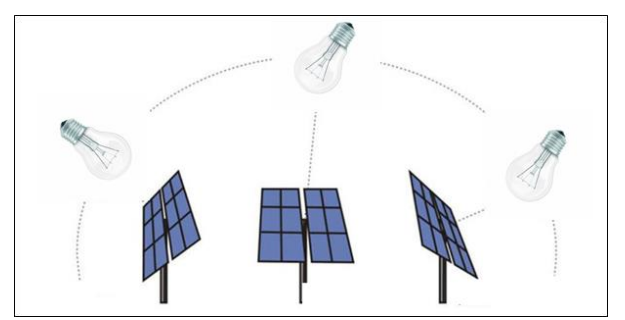

Fig. 9 Procedimento para Obtenção dos resultados. 


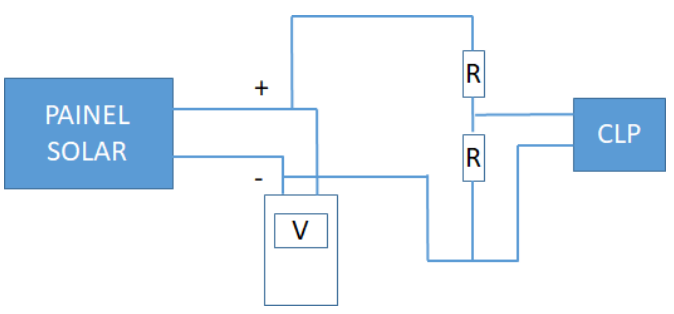

Fig. 10 - Ligações para leitura do valor gerado na saída do painel solar.

\subsection{Resultado do Experimento do Sistema de Controle}

O experimento foi realizado observando as seguintes ordens: 1- O painel foi deixado fixo à $90^{\circ}$, em seguida à $45^{\circ} \mathrm{e}$ por fim o controle implementado foi ativado. Para ambos os casos o caminho da fonte luminosa é o mesmo.

a) Painel Fixo à $90^{\circ}$

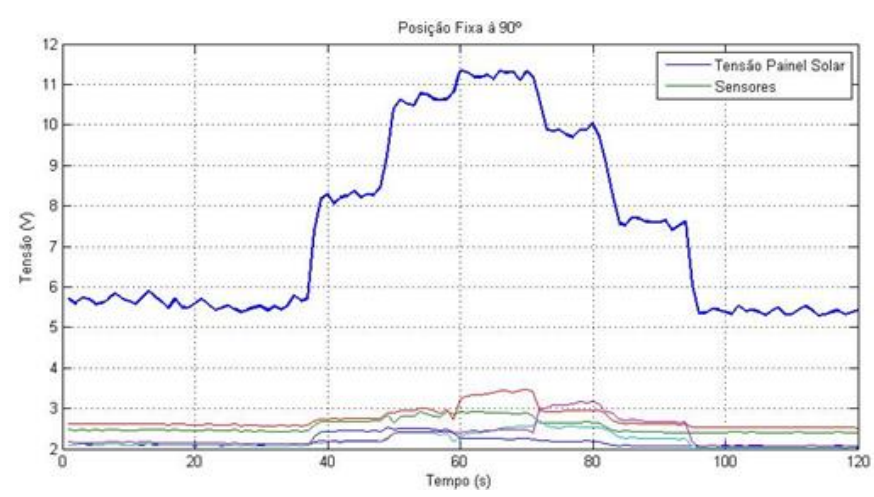

Fig. 11 Posição Fixa à 90

A Fig. 11 mostra a tensão gerada na saída do painel solar com o deslocamento da fonte luminosa. O comportamento do painel ao ser iluminado varia a tensão medida nos terminais, como esperado, a proporção que a fonte de luz se aproxima do ponto onde ficaria o mais perpendicular com o painel a tensão gerada aumenta.

b) Painel Fixo à $45^{\circ}$

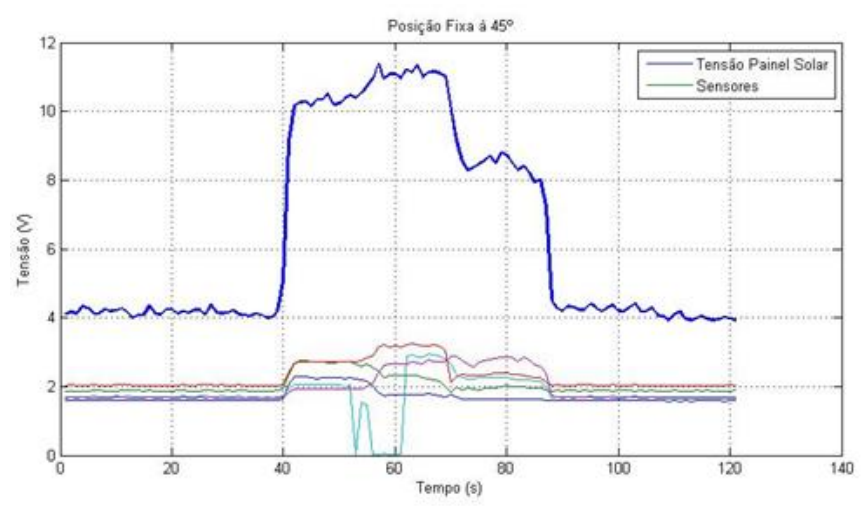

Fig. 12 Posição Fixa à 45
A Fig. 12 demonstra o comportamento da tensão gerada na saída do painel com o deslocamento da fonte luminosa. Percebe-se que quando a fonte de luminosa fica por trás do eixo do painel a geração diminui, como pode ser observado.

c) Painel com o Controle Implementado.

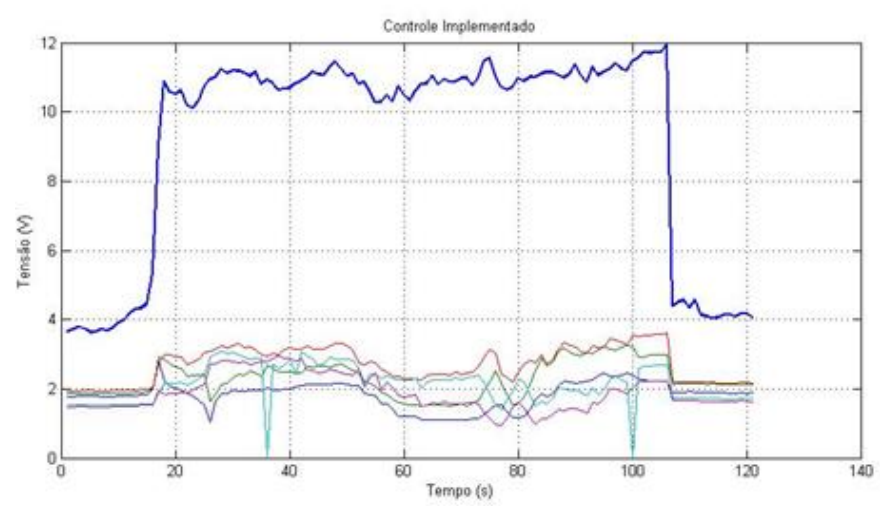

Fig. 13 Painel em movimento com a lógica implementada.

A Fig. 13 apresenta o controle implementado verifica- se que o valor da tensão lida é mantido em quase que semelhantes, vale ressaltar que a diferença está devido a distância e o próprio deslocamento da lâmpada que pode ter comprometido o valor da medição. No entanto, a Fig. 13 fica nítido o desempenho dos sensores e do controle implementado. Para este caso particular o painel iniciou em $90^{\circ} \mathrm{e}$ finalizou em $90^{\circ}$.

A análise deste experimento permite verificar a comparação da tensão lida na saída do painel fotovoltaico, bem como o trabalho dos sensores em função do deslocamento da fonte luminosa. Os resultados obtidos permitem a comparação do ganho em relação ao painel ficar fixo ou em movimento.

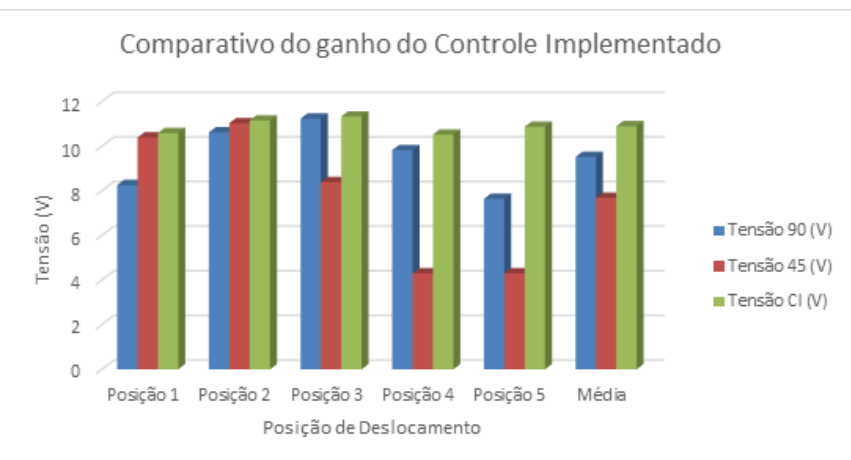

Fig. 14 Comparativo dos valores de tensão

A Fig. 14 apresenta o comparativo dos valores de tensão geradas na saída do painel quando este esteve fixo (à $90^{\circ}$ e à $45^{\circ}$ ) e quando o controle implementado permitiu que o painel se movimentar e acompanhar o deslocamento da fonte luminosa. Observa-se que em termos numéricos os resultados quando o controle foi implementado permitiu que o painel acompanhasse o movimento deixando o valor da tensão gerada quase sempre maior que nas demais posições.

Quando se analisa a partir da potência gerada pode-se fazer a análise a níveis de geração e gasto do controle implementado. 
Dessa forma, considera- se que a potência gasta para acionamento do motor em um segundo é $22,6 \mathrm{~mW}$, o tempo em média que o motor é acionado e parado é de $3 \mathrm{~s}$, logo em 3 segundos gastou- se $67,8 \mathrm{~mW}$ de potência. Estabelecendo que o tempo onde o controle será implementado, ou seja, a cada instante de tempo determinado, haverá uma atualização em relação ao valor das células, e assim o deslocamento do painel. Sendo este tempo 11 vezes, que significa que 11 vezes durante um dia por exemplo, haverá atualização, assim 11 vezes o motor será acionando gastando assim: $750 \mathrm{~mW}$ ou $0,75 \mathrm{~W}$ de potência. A corrente máxima gerada pelo painel é de 4,7A, tomando este valor e multiplicando pela média da tensão gerada nos terminais do controle implementando ter-se-ia uma potência gerada de $50,76 \mathrm{~W}$, descontando o valor perdido para acionamento dos motores ainda teria uma potência gerada de $50,01 \mathrm{~W}$. Com estes cálculos e discursões chega-se à conclusão que gastou-se um pouco mais de $1 \%$ para garantir a otimização da geração a partir de painéis fotovoltaicos.

\section{CONCLUSÃO}

Com os resultados experimentais comprova-se que o sistema tem uma maior geração quando o painel está perpendicular com o ângulo de incidência da fonte luminosa. Isto é visto quando se compara as tensões geradas nos experimentos realizados. O controle implementado mostrou-se eficiente na leitura dos valores medidos a partir das células possibilitando o tratamento dos dados e acionamento do motor para o movimento do painel. Além disso, o custo de implementação comparado com ganho da geração se mostrou aplicável, pensando-se em um investimento em escalas maiores.

É importante destacar que os resultados obtidos foram oriundos de um experimento realizado em laboratório, assim sendo para sua total aplicabilidade em meio externo são necessárias adaptações em algumas partes do projeto e testes para verificar a executabilidade. No entanto, os resultados apontam a efetividade do controle inteligente implementado.

\section{AGRADECIMENTOS}

Os autores querem agradecer a Universidade Federal Do Maranhão (UFMA), ao Departamento de Engenharia Elétrica (DEE), ao Instituto Federal do Maranhão (IFMA), ao Laboratório de Sistemas Integrados e Controle Inteligente (LABSECI), à Fundação de Amparo à Pesquisa e ao Desenvolvimento Científico e Tecnológico do Maranhão (FAPEMA) pelo auxílio financeiro e à ROCKWELL AUTOMATION pelos equipamentos fornecidos.

\section{REFERÊNCIAS}

FONSECA, J V, Ernesto F. M. F. (2013). Increase of PLC computability with neural network for recovery of fautls in electrical distribution substation. I2MTC: IEEE. p. 512

GALDINO, M A.E. et al. (2000). O Contexto das Energias Renováveis no Brasil. Rio de janeiro: Revista DIRENG. pp 1725 .
GOLDEMBERG, J and Chu, S (2004). Um futuro com energia sustentável: Iluminando o caminho. Tradução, Maria Cristina Vidal Borba, Neide Ferreira Gaspar. São Paulo: FAPESP, p.300.

KALOGIROU, S (2009). Solar energy engineering: process and systems. California: Elsevier Inc, p.476.

MASTERS, G M. (2004). Renewable and Efficient Electric Power Systems. New Jersey: John Wiley \& Sons, Inc, p. 445.

MIYAGI, P E. (2001). Controle Programável: Fundamentos do Controle de Sistemas a Eventos Discretos. $2^{\circ}$ Impressão. São Paulo: Editora Edgard Blucher LTDA, pp. 6 -12.

MORAES, C C and Castrucci, P. (2013). Engenharia de automação industrial. Rio de Janeiro: LTC Livros Técnicos e Científicos S. A.

RABELO, J E e R. (2017). Projeto em automação e controle: controle de sistemas a eventos discretos para posicionamento de painéis solares. São Luís: UFMA. pp 1 - 97. 